\title{
Observations upon the dynamic structure factor of interacting spherical polyelectrolytes ${ }^{\text {a) }}$
}

\author{
George D. J. Phillies \\ Department of Chemistry, University of Michigan, Ann Arbor, Michigan 48109 \\ (Received 17 January 1983; accepted 20 May 1983) \\ Experimental studies are reported on the light-scattering spectrum of $0.038 \mu$ carboxylate-modified \\ polystyrene latex spheres in deionized water. In concentrated suspensions, the long-time decay of the two- \\ particle dynamic structure factor is given by a power law $\left(t^{-a}\right)$ form, not by a slow exponential. Nonlinear \\ least squares fits found $\alpha \simeq 1.2-1.5$, smaller values of $\alpha$ corresponding to higher sphere concentrations. The \\ reduction of the mutual diffusion coefficient of $0.15 \mu$ spheres in mixtures of 0.15 and $0.038 \mu$ spheres was \\ examined, and is interpreted in terms of the dynamic friction modification to the drag coefficients. Multiple \\ scattering artifacts were shown to be absent with homodyne coincidence spectroscopy.
}

\section{INTRODUCTION}

Quasielastic light scattering has long been applied to the study of solutions of interacting monodisperse polyelectrolyte molecules. ${ }^{1,2}$ The concentration dependence of the slope $D_{\mathbb{M}} k^{2}$ of the initial decay of the dynamic structure factor $S(k, t)$ was early given theoretical interpretation in terms of a concentration-dependent friction factor $f$ and a thermodynamic factor related to the osmotic compressibility ${ }^{3}$ or the inverse of the static structure factor $S(k){ }^{4.5}$ Experimental confirmation of these equivalent theoretical interpretations was soon obtained. ${ }^{6-8}$ A careful examination of the form of $S(k, t)$ for nonideal macromolecule solutions revealed deviations at large $t$ from single-exponential decay. The deviations were first expressed ${ }^{2}$ in terms of the second cumulant of a cumulant expansion of $S(k, t)$; in other systems, these deviations have been described by a twoexponential model. ${ }^{9-11}$ A variety of interpretations of the long-time non-single-exponential behavior of $S(k, t)$ have been suggested, including memory function effects in the particle equations of motion, ${ }^{12}$ a time-dependent contribution of particle-particle interactions to the drag coefficient, ${ }^{13}$ concentration-concentration mode-mode coupling terms in the diffusion equation, and polydispers ity in either the interparticle interactions ${ }^{14}$ or the particle sizes. 15,16

We here report experimental studies on the light scattering spectra of suspensions of polystyrene latex spheres in deionized water. Our major objectives included a more careful study of the functional form of $S(k, t)$ at very long times, a study of multiple scattering effects with a homodyne coincidence spectrometer, ${ }^{17}$ and work on mixtures containing interacting polystyrene spheres of known, different sizes.

Experimental methods are treated in Sec. II. Experimental results are presented in Sec. III and inter preted in Sec. IV. Section V contains a discussion and summary.

\section{METHODS}

Studies were made of carboxylate-modified polystyrene latex spheres of nominal diameters $0.038 \mu$ (Dow Pharm-

\footnotetext{
${ }^{2}$ The support of this work by the National Science Foundation under Grant CHE79-20389 is gratefully acknowledged.
}

aceuticals) and $0.15 \mu$ (Polysciences, Inc.). Light scattering spectra of dilute suspensions of these spheres in distilled nondeionized water found radii of 220 and $840 \AA$, respectively. Deionized sphere suspensions were prepared in quartz fluorimeter curvettes, four sides polished, by diluting known volumes of the sphere suspensions with 15 Ms distilled water, adding specially cleaned mixed-bed ion-exchange resin, and aging for three to six weeks. It was at first found that samples deteriorated after several months, their spectra returning towards the forms observed before the ion-exchange resin was added. It was suspected that this effect was due to the slow leakage of $\mathrm{CO}_{2}$ around the Teflon stoppers of the fluorimeter cuvettes. Coating the stopper rim, not at points in contact with the sample, with high-vacuum stopcock grease put an end to the progressive deterioration.

Spectra were taken of the $0.038 \mu$ spheres at nominal sphere volume fractions of $1 \times 10^{-4}, 5 \times 10^{-4}, 1.3 \times 10^{-3}$, $2.5 \times 10^{-3}, 3.8 \times 10^{-3}$, and $7.5 \times 10^{-3}$. Spectra were also obtained by mixtures of the 0.038 and $0.15 \mu$ spheres, using the aforementioned concentrations for the $0.038 \mu$ spheres, together with the $0.15 \mu$ spheres at a nominal concentration of $6 \times 10^{-5}$ by volume.

Our light scattering spectrometer incorporates a 25 $m W$ HeNe laser, rigid optics based on a Newport Research vibration-isolation table, RCA 7265 photomultiplier tube coupled to Pacific Precision amplifier discriminator, and a 64 channel Langley-Ford Instruments digital correlator interfaced to a Data General Nova III computer. To obtain high resolution at short times and good signal-to-noise ratios at long times, it was necessary to make measurements on each sample using a ser ies of different integration times and correlator channel spacings; overlapping spectra were then spliced. Several different splicing methods were tested, including (i) forcing all spectra to have a common base line (measured by delay channels in the correlator) and a common amplitude at a time $t$ at which the signal-tonoise ratio of $S(k, t)$ was high, (ii) forcing agreement be tween spectral amplitudes at two well-separated delay times, using the base lines as free parameters, and (iii) graphical overlay and optical line fitting. All these methods give very similar results, though the optical method is modestly less precise than the alternate digi- 


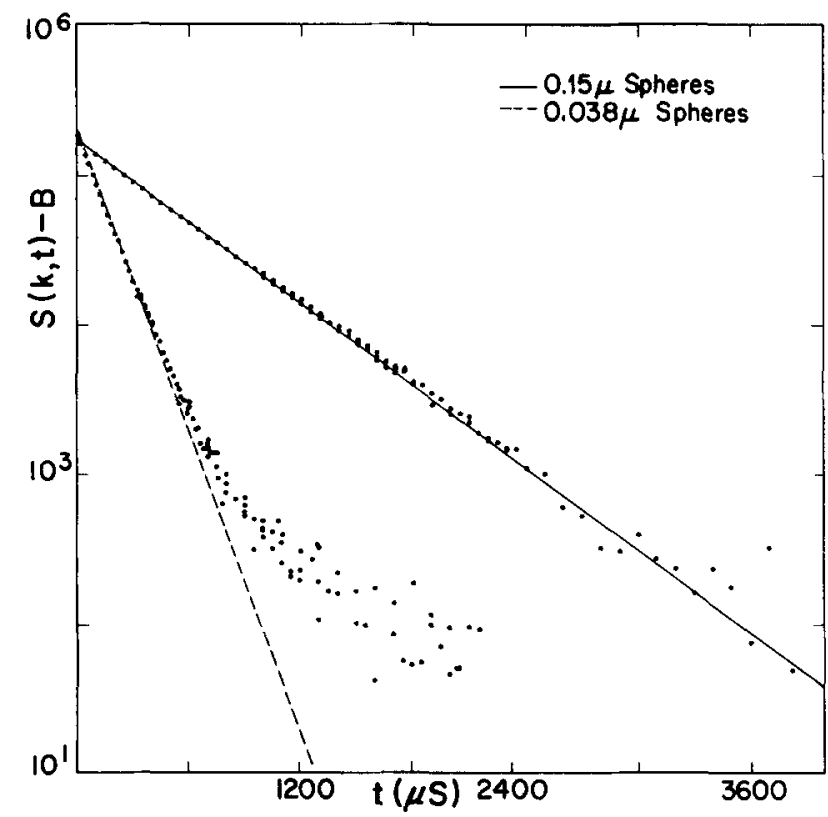

FIG. 1. Light scattering spectra of 0.038 (line, dots) and $0.15 \mu$ (line, dots) carboxylate-modified polystyrene latex spheres in pure water, prior to removal of trace ions from the water. Sphere concentrations were $C_{0_{0} 038}=0.02$ volume \%, $c_{0.15}=6 \times 10^{-3}$ volume $\%$.

tal procedures. Error estimates were obtained by comparing results on single unspliced spectra with results on the merged spectra.

Spectra were fit to two functional forms. To obtain the limiting initial slope of $S(k, t)$ as $t \rightarrow 0$, and to study linear segments of more-complex spectra, we analyzed light scattering spectra, or their long or short time segments, by linear-least squares fit to a cumulant expansion

$$
\frac{1}{2} \ln [S(k, t)-B]=\sum_{i=0}^{n} \frac{K_{1}(-t)^{t}}{i !},
$$

$B$ being the base line and $K_{1}$ being the $i$ th cumulant. The long-time sections of spectra were also subject to the nonlinear least squares fit, using an optimized twoparameter mesh-scanning program, the assumed longtime form of the spectrum being

$$
S(k, t)=a_{0} t^{-b} 0+B \text {. }
$$

The base line was determined experimentally. Multiple scattering effects were tested by means of the homodyne coincidence spectrometer described in Ref. 17.

\section{RESULTS}

By using multihour integration times, it was possible to obtain reasonably accurate measurements of the form of the spectrum $S(k, t)$ over several decades of its decay. Apparent signal-to-noise ratios near $t=0$ of the order $1 \times 10^{3}$ were obtained. Control experiments in which the spectra of nondelonized sphere suspensions were measured with multihour integration times gave the expected spectra. Even with a multihour integration time, the spectrum of a constant signal (a piece of lens paper il- luminated by a flashlight) was found to be essentially flat. The nonexponential spectra reported here for more concentrated systems are therefore interpreted as arising from physical effects in the sample, and not from laser noise or other artifacts.

Figure 1 shows spectra of the 0.038 and $0.15 \mu$ carboxylate-modified polystyrene latex spheres, at low concentration, in distilled water to which ion-exchange resin had not been added. To within experimental error, the spectrum of the $0.15 \mu$ spheres exhibits a single exponential curve over three orders of magnitude in the decay of $S(k, t)$. In particular, the exponential decay appears entirely linear in the time range (1-3 ms) and amplitude region $[0.1 \%-10.0 \%$ of $S(k, 0)]$ in which interesting effects are seen in the spectra of the concentrated deionized suspensions. The spectrum of the $0.038 \mu$ spheres at low concentrations in untreated water does show a weak deviation from a single exponential. This deviation is not pronounced for $S(k, t)>0.003 S(k, 0)$.

The effect of removing the background of small ions from the solvent is shown in Fig. 2. In this figure, one sees spectra of $0.038 \mu$ polystyrene sphere suspensions of concentrations $0.05 \%, 0.25 \%$, and $0.75 \%$ by volume. These suspensions were thoroughly deionized with specially cleaned ion exchange resin.

The spectrum of the $0.05 \%$ solution is consistent with a simple two-exponential model. Indeed, there are clear systematic differences between a power-law fit to this spectrum [Fig. 2(a), solid line] and the experimental data. In contrast, at high concentration, the spectrum is nonexponential at long times. In considering the $0.25 \%$ suspension, if one were to limit one's self to times $<1500 \mu \mathrm{s}$, the "long-time" part of $S(k, t)$ could be said to be a slow exponential, as indicated by the dashed line of spectrum 2(b). However, at times larger than $1800 \mu \mathrm{s}$, the amplitude of the measured spectrum (dots) is visibly too large to be consistent with the slow exponential characterization. One could use additional, even slower, exponentials to describe the spectrum at these very long times, but it would be very hard to determine such exponentials in a meaningful way. Alternately one could impose a cutoff in time, and only fit $S(k, t)$ for times less than cutoff value, but exponentials obtained in this way would be artifacts of cutoff time.

In concentrated suspensions, mode-mode coupling ef fects may be significant, motivating consideration of the possibility that the long-time decay of $S(k, t)$ is described by a power law rather than an exponential. The hypothesis that $S(k, t)$ shows a long-term power law decay was tested by making a nonlinear least squares fit of $S(k, t)$, for $t>900 \mu \mathrm{s}$, to Eq. (2). Separate fits were made to each spectrum obtained on each sample. A simultaneous weighted fit of Eq. (2) to all the spectra on a given sample, using $S(k, 1 \mathrm{~ms})$ of each spectrum as a weighing factor, was also made. The solid lines in Fig. 2 are the results of a simultaneous fit to all data. Unlike the dashed lines, the solid lines appear to be reasonable representations of $S(k, t)$ for concentrated suspensions at large time. 


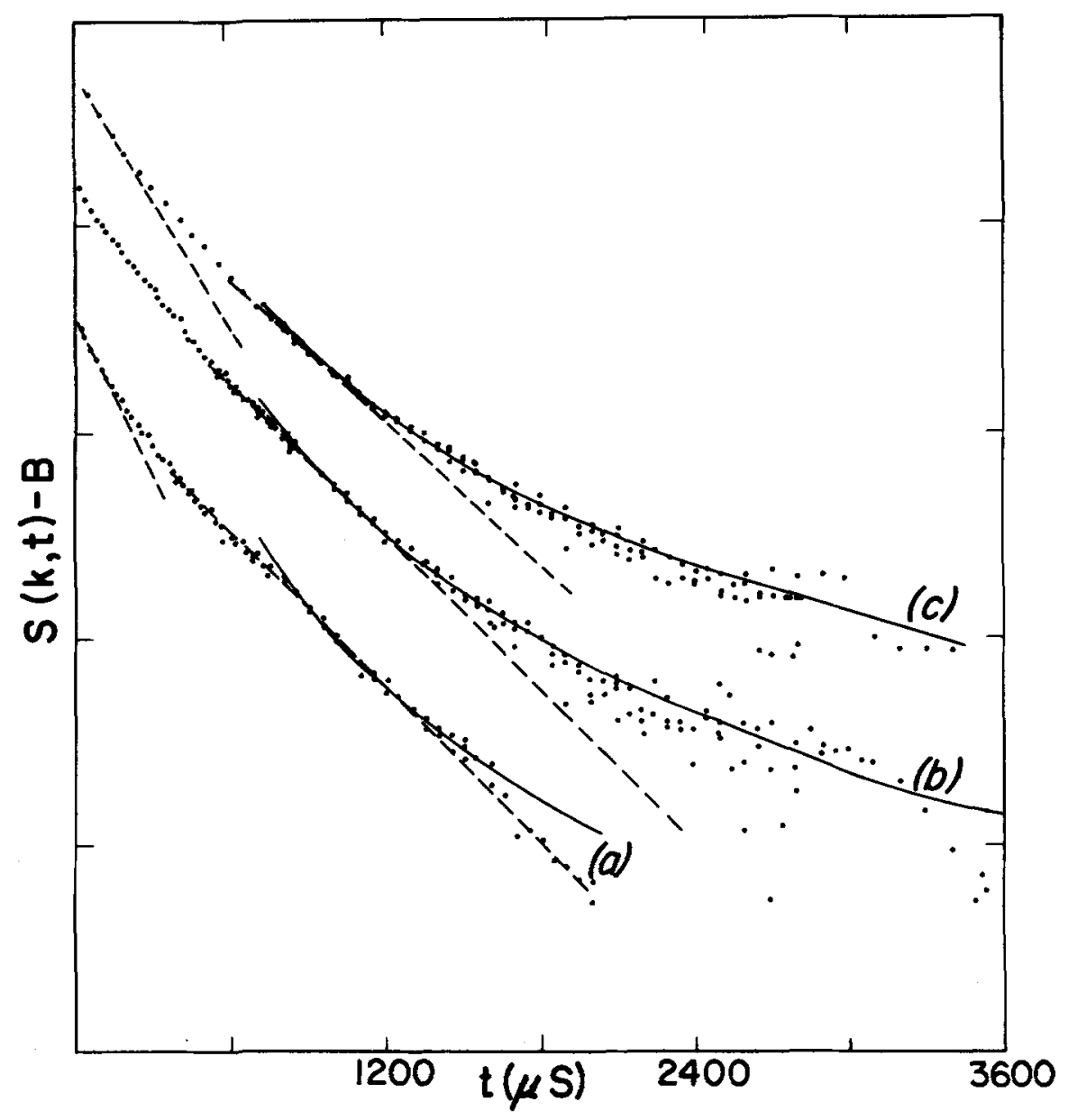

FIG. 2. Spliced light-scattering spectra $S(k, t)$ of $0.038 \mu$ polystyrene spheres at concentrations of (a) 0.02 , (b) 0.25 , and (c) 0.75 volume $\%$, after removal of small ion contaminants with lon-exchange resin. The amplitude units are arbitrary. The dashed straight lines indicate the single-exponential decays which match limited regions of the spectra; solid curved lines indicate nonlinear least-mean-square fits of the spectra (for $t>900 \mu_{\mathrm{S}}$ ) to a power law [Eq. (2)].

Figure 3 displays the decay coefficient $b_{0}$ of the longtime component of $S(k, t)$, as a function of the sphere concentration. $b_{0}$ decreases from $3.2+/-0.1$ at a sphere concentration of $0.13 \%$ to $2.45+/-0.1$ at a sphere concentration of $0.75 \%$. At sphere concentrations below $0.1 \%$, the form of $S(k, t)$ seems to be inter mediate between a slow exponential and a power law. At these concentrations, one can still fit the long-time part of $S(k, t)$ to a power law decay, but deviations of the spectrum from the power-law form are systematic. $b_{0}$ for these concentrations was therefore not plotted in Fig. 3.

Several authors ${ }^{15,16}$ discuss the possibility that the long-time tail of $S(k, t)$ may be due to scattering polydispersity in the sample. Implicit in these models is the assumption that diffusional modes which exchange particles of differing scattering power ("tracer"-like models) are substantially hindered by direct and hydrodynamic interactions between the diffusing particles. To test this suggestion, mixture s containing a combination of $0.038 \mu$ spheres (at the concentrations used in the abovementioned experiments) and $0.15 \mu$ spheres (at a concentration of $6 \times 10^{-5}$ by volume) were studied. As seen in Fig. 4, spectra of these samples show two clear exponentials. The faster, weak, exponential is not distinguishable in form from the decay spectrum of the $0.038 \mu$ spheres in the absence of $0.15 \mu$ spheres, though because of its low relative amplitude this weaker decay can only be studied at relatively short times $(<600 \mu \mathrm{s})$. The dominant relaxational mode of the system is a slow single exponential whose decay time is similar to but less than that of the $0.15 \mu$ spheres in pure

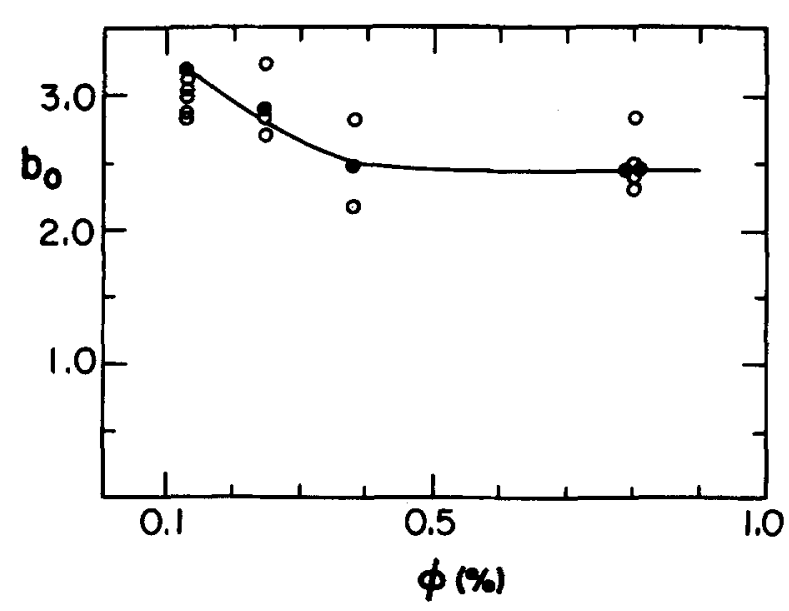

FIG. 3. Dependence of the power-law-decay coefficient $b_{0}$, obtained by fitting spectra to Eq. (2), on the sphere concentration. Filled dots indicate fits to spliced spectra such as those shown in Fig. 2; open dots indicate fits of Eq. (2) for individual spectra. At very low concentrations, Eq. (2) is not a good representation of the long-time tail of $S(k, t)$ [Fig. 2(a) presents an example]; $b_{0}$ for these low concentrations has not been plotted. 


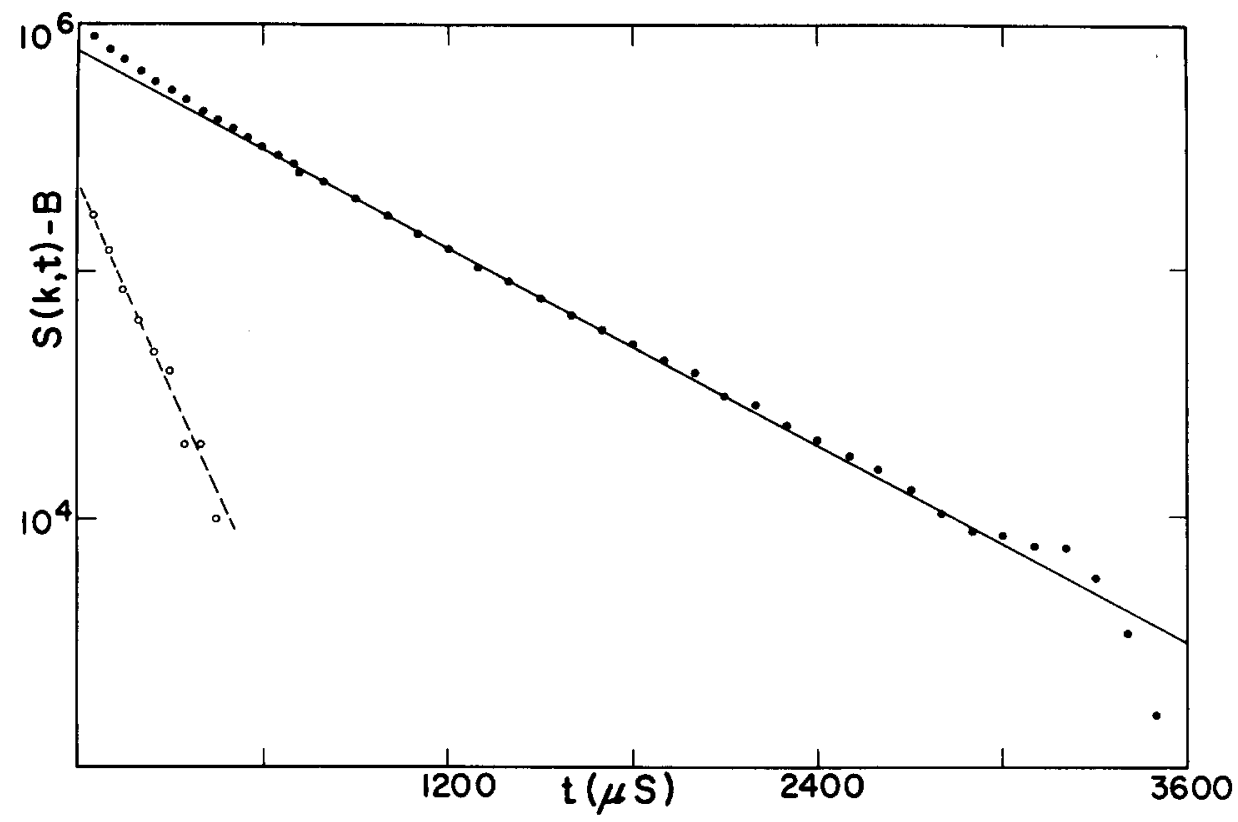

FIG. 4. Light scattering spectrum of a deionized mixture of 0.038 and $0.015 \mu$ polystyrene spheres at concentrations of 0.38 and 0.006 volume $\%$, respectively. The straight line is the long-time single exponential fit used to determine the diffusion coefficient $D_{T}$. The inset is the difference between the observed spectrum and a single slow exponential; this difference spectrum is not noticeably different in form from the spectrum of deionized $0.038 \mu$ spheres at the same concentration.

water. We denote the diffusion coefficient deduced from this slow decay $D_{T}$.

Figure 5 shows $D_{T}$ as a function of the concentration of the smaller $(0.038 \mu)$ spheres. Even at very high concentrations of the smaller spheres, direct measurement shows that the small spheres by themselves scatter much less light than the mixtures do, so the spectra are due primarily to scattering by the larger spheres. At very low small-sphere concentrations, $D_{T}$ approaches the diffusion coefficient of the larger spheres in pure water. $D_{T}$ falls with increasing small-sphere concentration. If one naively interprets $D_{T}$ as arising from the motion of the larger spheres through the smaller, then, over the concentration range studied, the small spheres reduce the diffusion coefficient of the larger spheres by as much as $30 \%$.

Polystyrene latex has an index of refraction increment which is substantially different from that of water. At higher sphere concentrations $S(k, t)$ might therefore be perturbed by multiple scattering. Since our objective is to study concentration effects, an extrapolation of data to zero concentration would not be helpful. Recourse was instead made to homodyne coincidence spectroscopy, ${ }^{17}$ a two-laser-beam, two-detector method for obtaining the single-scattering contribution to $S(k, t)$. As has previously been shown, homodyne coincidence instruments are immune to multiple scattering artifacts. Because our homodyne cross-correlation efficiency is of order $10 \%$, our measurements of $S(k, t)$ at large $t$ were appreciably less accurate with two detectors than with one detector. For small $t$, accurate measurements of the initial slope of $S(k, t)$ on deionized samples with homodyne coincidence are in good agreement with one-detector spectra, indicating that multiple scattering (which is expected to be most important at small $t$ ) is not significant. The absence of multiple scattering artifacts is consistent with the observation that the samples discussed in this paper are markedly less turbid than the multiply scattering solutions studied in Ref. 17.

The scattering vector $|\mathrm{k}|$ in this experiment was 1.87 $\times 10^{5} \mathrm{~cm}^{-1}$, i. e., $|\mathrm{k}|^{-1}=535 \AA$. For the $1 \times 10^{-4}, 5 \times 10^{-4}$, $1.3 \times 10^{-3}, 2.5 \times 10^{-3}, 3.8 \times 10^{-3}$, and $7.5 \times 10^{-3}$ volume fraction solutions, the mean interparticle distances may be estimated as $8800,5100,3700,3000,2600$, and 2100 $\AA$, respectively, so that in this experiment particle motions were observed over distances short by comparison with the mean interparticle distance. A superficial examination of the scattered light intensity of angle revealed no sign of a maximum in the scattered intensity $S(k)$, but this phenomenon would not necessarily have been expected in our relatively dilute solutions.

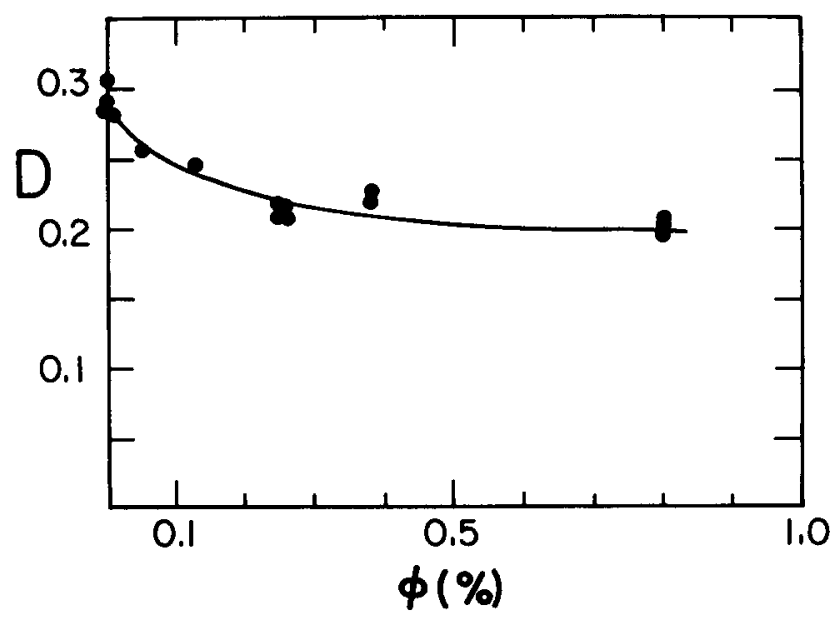

FIG. 5. Dependence of the diffusion coefficient $D_{T}$ obtained from spectra of the $0.038 / 0.15 \mu$ sphere mixtures on the concentration of the smaller spheres, the concentration of the larger spheres being held fixed. Also shown is the diffusion coefficient of the $0.15 \mu$ spheres in a deionized solution in the absence of any $0.038 \mu$ spheres. Diffusion coefficients are in units $10^{-7} \mathrm{~cm}^{2} / \mathrm{s}$. 


\section{INTERPRETATION}

Our first objective was to study the functional form of $S(k, t)$ at long times. As our spectra decrease monotonically and are convex upwards, they may presumably be represented, with good accuracy, as a sum of exponentials; Grüner and Lehman have used this approach. This paper treats an alternate representation for $S(k, t)$. Just as the two-exponential approximation for $S(k, t)$ suggests several ${ }^{11,18}$ theoretical models for these solutions, alternate representations of $S(k, t)$-such as the powerlaw form successfully tested here-may suggest other plctures of a macroparticle suspension.

Our scattering volume was much larger than the static and dynamic correlation ranges of the system, so the central limit theorem indicates that the scattering intensity is a Gaussian random process. $S(k, t)$ is then related to the density $\rho_{k}(t)$ of scatterers by

$$
\begin{aligned}
S(k, t)-B & =\left|\left\langle\sum_{i, j=1}^{N} b_{i} b_{j} \exp \left\{i \mathrm{k} \cdot\left[\mathbf{r}_{i}(0)-\mathbf{r}_{j}(t)\right]\right\}\right\rangle\right|^{2} \\
& \equiv\left|\left\langle\rho_{k}(0) \rho_{-k}(t)\right\rangle\right|^{2},
\end{aligned}
$$

$B$ being the base line, $b$, and $r_{j}(t)$ being the scattering cross section and the position at time $t$ of the scatterer $j$, and $\rho_{k}$ being the $k$ th spatial Fourier component of the scatterer density.

$S(k, t)$ was found to be concentration dependent, passing from one exponential at extreme dilution to a two-exponential form at low sphere concentrations and a power-law decay at higher concentrations. From Eq. (3), the concentration-concentration correlation function $\left\langle\rho_{k}(0) \rho_{-k}(t)\right\rangle$ must also be described by a two-exponential form at low concentration and by a power-law $\left(t^{-1.2}\right.$ to $\left.t^{-1.6}\right)$ decay at high concentration and large $t$. The similarity between our high concentration data and the theoretical long-time $t^{-3 / 2}$ decays, characteristic of mode-coupling effects, suggests but does not prove that concentration-concentration mode coupling effects are important.

Theories for $S(k, t)$ for strongly interacting suspensions are largely not helpful in understanding our results. Most work has emphasized the first time cumulant of $S(k, t)$ in systems sufficiently dilute that only inieractions between isolated pairs of particles are significant. At least two calculations ${ }^{19,20}$ of the second time cumu lant of $S(k, t)$ have been made, albeit for a model system without hydrodynamic interactions. While either of these inequivalent calculations can be iterated to obtain higherorder time cumulants, Interpreting our data would require an unreasonably great number of iterations. Furthermore, the observed power-law decay is not experimentally correct at short times (indeed, it is not analytic at $t=0$ ), so an expansion of $S(k, t)$ as a Taylor series around $t=0$ can only give numerical agreement with our findings. In addition to these difficulties, modecoupling terms involve the effects of interacting trios of particles, i.e., they are only important at second and higher orders in the concentration. The published calculations ${ }^{21,22}$ of the $0\left(C^{2}\right)$ corrections to $D$ are only for the initial slope or the low-frequency limit of $D$.

Several author ${ }^{18,23}$ have used the $\mathrm{Zwanzig-Mori}$ formallsm to calculate $S(k, t)$. The difficult part of these computations is finding a form for the Mori memory kernel $M(k, t)$. For example, if $M(k, t)$ is an exponential, $S(k, t)$ is a double exponential. Forms for $M(k, t)$ resulting in $S(k, t) \sim t^{-\alpha}$ at large $t$ seem not to have been treated.

The data represented by Figs. 1-3 does not conclus ively determine whether the long-time tail of $S(k, t)$ is due to interactive effects or to polydispersity. Cur rent theories of interacting suspensions do not treat $S(k, t)$ at long times; while there are plausibility arguments, a quantitative comparison of experiment and prediction is presently impossible. Published work ${ }^{15,24}$ on polydispersity predicts a long time slow exponential, not a $t^{-\alpha}$ form, but this is clearly an artifact of the approximations used in that work. If a solution contained trace quantities of particles having a range of sizes, the solutions's spectrum would exhibit a range of slow exponentials, whose sum could readily mimic a power-law decay. Our results therefore do not disprove the conjecture that the $t^{-\alpha}$ tail is caused by polydispersity.

Our second objective was to study scattering from bidisperse suspensions. Weissman's proposal $1^{15}$ on polydispersity effects gave one motivation for this work. Arguments ${ }^{13}$ that dynamic friction (which results from the correlation between the Brownian movements of a particle and the subsequent interparticle forces on that particle) hinders both mutual and self-diffusion gave another motivation.

The scattering spectrum of a solution contalning two interacting macroparticle species was computed a decade ago. ${ }^{25}$ Pusey $^{24}$ and this author ${ }^{26}$ have treated the special case in which the two species are identical except for their scattering cross sections. The bidisperse suspensions reported here for $m$ an alternate special case, in which the scattering is dominated by a species whose concentration is vanishingly small. As shown in Appen$\operatorname{dix} A$, if one ignores mode-coupling terms the scattering spectrum for this special case is

$$
\begin{aligned}
{[S(k, t)-B]^{1 / 2}=} & \exp \left(-D_{\mathrm{A}} k^{2} t\right)\left\{I_{\mathrm{A}}-\frac{D_{\mathrm{BA}}}{D_{\mathrm{B}}-D_{\mathrm{A}}} I_{\mathrm{AB}}\right\} \\
& +\exp \left(-D_{\mathrm{B}} k^{2} t\right)\left\{I_{\mathrm{B}}+\frac{D_{\mathrm{BA}}}{D_{\mathrm{B}}-D_{\mathrm{A}}} I_{\mathrm{AB}}\right\} .
\end{aligned}
$$

Here $A$ is the dilute, intensely scattering species, $D_{A}$ is the self-diffusion coefficient of $A$ through the $A-B$ mixture, $D_{B}$ is the mutual diffusion coefficient of $B$ in the absence of $A$, and $I_{A}$ and $I_{B}$ are the scattering intensities due to species $A$ and $B$, respectively, including interference effects due to correlations between molecules of the two species. The $I_{A B}$ terms results from a dynamic effect in which the cross-diffusion coefficient $D_{B A}$ (which gives the current in $B$ due to a concentration gradient of A) transfers intensity from one relaxational mode to the other; $I_{A B}$ vanishes if either species scatters no light.

Equation (4) justifies our interpretation of Figs. 4 and 5 by predicting that the dominant slow exponential describes the self-diffusive motion of the highly dilute 0.15 $\mu$ spheres (" $A$ " component) through a concentrated background of more weakly scattering $0.038 \mu$ spheres (" $B$ " component). Equation (4) predicts that the weak, rapid- 
ly decaying exponential of the mixtures should match the spectrum of the $0.038 \mu$ spheres in the absence of $0.15 \mu$ spheres. This was found experimentally. Equation (4) is based on a linear two-component theory so it can not predict long-time tails, regardless of one's model for them. Since the long-time decays of Fig. 2 would be too weak to see in Fig. 4, and deionized $0.15 \mu$ spheres at $\phi \approx 6 \times 10^{-5}$ have a single-exponential spectrum, the absence of long-time nonexponential effects in Fig. 4 is unsurprising.

As seen in Fig. 5, the $0.038 \mu$ spheres act to reduce the self-diffusion coefficient $D_{T}$ of $0.15 \mu$ spheres in mixtures. Mazur and vanSaarloos ${ }^{27}$ have recently obtained the 2,3, and 4-point hydrodynamic interaction tensors for spheres of different sizes, so the expected hydrodynamic effect of the smaller spheres on the motions of the larger ones is readily obtained (cf. Appendix B) as the approximation:

$$
D_{T}=D_{15}^{0}\left(1-\frac{15}{4} \phi_{38}\left[\frac{a_{15}}{\sigma_{15}+\sigma_{38}}\right]\right),
$$

$D_{15}^{0}$ being the diffusion coefficient of an isolated $0.15 \mu$ sphere, $\phi_{38}$ being the volume fraction of the $0.038 \mu$ spheres (in our mixtures, $\phi_{38}<0.01$ ), $a_{15}$ being the hydrodynamic radius of the $0.15 \mu$ particles, and $\sigma_{15}$ and $\sigma_{38}$ being the radil of exclusion of the 0.15 and 0.038 $\mu$ particles. To obtain Eq. (5), the charged hard spheres were approximated as neutral hard spheres with an enhanced distance of closest approach $\sigma_{15}+\sigma_{38}$, $\sigma_{15}+\sigma_{38}$ being larger than the hydrodynamic diameter $a_{15}+a_{38}$. The effect of charging is to reduce the hydrodynamic correction in Eq. (5); if the spheres were neutralized, the effect of hydrodynamics on $D_{T}$ would be increased. Equation (5) predicts a dependence of $D_{T}$ on $\phi_{38}$ which is much less than the apparent initial slope visible in Fig. 5, so the dependence of $D_{T}$ on $\phi_{38}$ is largely not due to hydrodynamic interactions between the larger and smaller spheres.

One explanation of the concentration dependence of $D_{T}$ is that the motion of the larger spheres is hindered by dynamic friction, due to the fluctuating forces exerted on the larger spheres by the smaller ones. It has been shown theoretically ${ }^{13}$ that such fluctuating forces between diffusing macroparticles enhance the friction factors $f$ for mutual and tracer diffusion, so this effect has the right sign to account for the failure of Eq. (5). Indeed, Hess and Klein, ${ }^{28}$ beginning with a Focker-Planck equation, recently used projection operators and a modemode coupling formalism to obtain the modification of the self-diffusion coefficient of a charged sphere by direct interactions. The calculation, which neglects hydrodynamic interactions, uses the static structure factor measured by Grüner and Lehmann. ${ }^{11}$ Hess and Klein obtain for the concentration dependence of $D_{s}$ a plot qualitatively identical to Fig. 5 .

\section{DISCUSSION}

It is perhaps surprising that we find a $t^{-\alpha}$ form for $S(k, t)$ at large $t$, rather than the slow exponential reported by others. If Weissman's polydispersity interpretation is correct, the variations in $S(k, t)$ may sim- ply reflect differences in the macroparticle size distribution function and thus may not be physically important. Furthermore, unless one goes to extremely large times, a power law decay looks much like an exponential; with some spectra, power-law and exponential fits will both be qualitatively acceptable. Contrariwise, to show that one does have a single slow exponential, and not a powerlaw decay, one must obtain high-quality data for times appreciably greater than those needed to calculate the apparent decay constant of the exponential.

The diffusion of very dilute $0.15 \mu$ spheres is retarded by $0.038 \mu$ spheres, the extent of the retardation being much greater than that expected from the hydrodynamic interactions between the spheres. Hess and Klein, ${ }^{28}$ us ing an equation substantially identical to our for $\mathrm{m}^{13}$ for the dynamic friction effect on the diffusion coefficients, calculated the concentration dependence of a quantity similar to $D_{T}$. While some details are different, the qualitative agreement between their theoretical numbers and our data is excellent.

The diffusion of interacting macroparticles of differing sizes and scattering cross sections has been extensively studied by Kops -Werkhoven et al. ${ }^{29}$ who obtain results in many respects similar to those reported here. In their system, which was a suspension of neutral hard spheres, increasing the concentration (up to 50 volume $\%)$ resulted in two-exponential spectra and in changes in the mutual and tracer diffusion coefficients of the spheres. The differences in $S(k, t)$ in between Ref. 29 and the results here could be due to the long range of the forces between charged spheres.

\section{ACKNOWLEDGMENT}

I wish to thank Dr. Alan Hurd for his gift of pretreated ion exchange resin.

\section{APPENDIX A: LIGHT-SCATTERING SPECTRA OF TWO-INTERACTING MACROCOMPONENT MIXTURES}

Reference (25) obtains the quasielastic light scattering spectrum of a three-component (solvent, solute A, solute $B$ ) system whose diffusion obeys the linear flow equations

$$
\begin{aligned}
& \frac{d}{d t} a_{\mathrm{A}}(k, t)=-\Gamma_{\mathrm{A}} a_{\mathrm{A}}(k, t)-\Gamma_{\mathrm{AB}} a_{\mathrm{B}}(k, t), \\
& \frac{d}{d t} a_{\mathrm{B}}(k, t)=-\Gamma_{\mathrm{BA}} a_{\mathrm{A}}(k, t)-\Gamma_{\mathrm{B}} a_{\mathrm{B}}(k, t),
\end{aligned}
$$

where

$$
\Gamma_{\mathrm{A}}=D_{\mathrm{A}} k^{2}, \quad \Gamma_{\mathrm{B}}=D_{\mathrm{B}} k^{2}, \quad \Gamma_{\mathrm{AB}}=D_{\mathrm{AB}} k^{2}, \quad \Gamma_{\mathrm{BA}}=D_{\mathrm{BA}} k^{2},
$$

$k$ is the scatter ing vector, and $a_{1}(k, t)$ is the amplitude of the $k$ th spatial Fourier component of the concentration of $i$. The spectrum of this solution is predicted to be

$$
\begin{aligned}
& \langle I(0) I(t)\rangle-B=I_{0} A^{-1}\left(\Gamma^{+}-\Gamma^{-}\right)^{-1}\left(e ^ { - \Gamma ^ { + } t } \left\{\left(\Gamma_{A}-\Gamma^{-}\right)\left(\epsilon_{a}^{2} \alpha+\epsilon_{a} \epsilon_{b} \gamma\right)\right.\right. \\
& \left.+\left(\Gamma^{+}-\Gamma_{A}\right)\left(\epsilon_{b}^{2} \beta+\epsilon_{a} \epsilon_{b} \gamma\right)+\Gamma_{A B}\left(\epsilon_{a}^{2} \epsilon_{b} \alpha+\epsilon_{b}^{2} \gamma\right)\right\} \\
& +e^{-\Gamma^{-} t}\left\{\left(\Gamma^{+}-\Gamma_{A}\right)\left(\epsilon_{a}^{2} \alpha+\epsilon_{a} \epsilon_{b} \gamma\right)+\left(\Gamma_{A}-\Gamma^{-}\right)\left(\epsilon_{a} \epsilon_{b} \gamma+\epsilon_{b}^{2} \beta\right)\right. \\
& \left.\left.\quad-\Gamma_{A B}\left(\epsilon_{a}^{2} \gamma+\epsilon_{a} \epsilon_{b} \beta\right)-\Gamma_{B A}\left(\epsilon_{a} \epsilon_{b} \alpha+\epsilon_{b}^{2} \gamma\right)\right\}\right),
\end{aligned}
$$




$$
\begin{aligned}
& \Gamma^{ \pm}=\frac{1}{2}\left(\Gamma_{\mathrm{A}}+\Gamma_{\mathrm{B}}\right) \pm\left\{\left[\frac{1}{2}\left(\Gamma_{\mathrm{A}}-\Gamma_{\mathrm{B}}\right)\right]^{2}+\Gamma_{\mathrm{AB}} \Gamma_{\mathrm{BA}}\right\}^{1 / 2}, \\
& a_{0}=a_{\mathrm{A}}(k, 0), \\
& b_{0}=a_{\mathrm{B}}(k, 0) . \\
& A=\epsilon_{a}^{2} \alpha+2 \epsilon_{a} \epsilon_{b} \gamma+\epsilon_{\mathrm{b}}^{2} \beta, \\
& \alpha=\left\langle\left|a_{\mathrm{A}}(k, 0)\right|^{2}\right\rangle, \\
& \beta=\left\langle\left|a_{\mathrm{B}}(k, 0)\right|^{2}\right\rangle, \\
& \gamma=\left\langle\left|a_{\mathrm{A}}(-k, 0) a_{\mathrm{B}}(k, 0)\right|\right\rangle .
\end{aligned}
$$

$\epsilon_{A}$ and $\epsilon_{B}$ being the optical scattering lengths (the cross sections being $\epsilon_{A}^{2}, \epsilon_{B}^{2}$ ) for the two species.

In our system, the strong interactions arise from the great range of the forces. The hydrodynamic volume of either species is small, so reference frame corrections ${ }^{30}$ are negligible. This approximation would be poor for $\phi \geqslant 10 \%$. From Ref. 25, Sec. 4, Ref. 3, Sec. 4 and an integration by parts, the diffusion coefficients are

$$
D_{i j}=\frac{K_{B} T\left[\delta_{i j}+c_{i} h_{i j}(k)\right]}{f_{i}},
$$

where $\delta_{i j}$ is a Kronecker delta, $f_{i}$ is the drag coefficient of species $i$ in the solution of interest, $c_{i}$ is the concentration of $i$, and $h_{i j}(k)$ is the spatial Fourier transform of the $i-j$ radial distribution function.

In our special case, $D_{B}>D_{A}, \epsilon_{\mathrm{A}}>\epsilon_{\mathrm{B}}, N_{A} \epsilon_{A}^{2} \gg N_{B} \epsilon_{B}^{2}$ (but the scattering by $\mathrm{B}$ does not vanish, so $N_{B} \epsilon_{B}^{2}>0$ ), and $C_{\mathrm{A}} \approx 0$. On neglect of terms in $C_{\mathrm{A}}, \mathrm{Eq}$. (A2) reduces to Eq. (4), where

$$
\begin{gathered}
I_{\mathrm{A}}=\epsilon_{\mathrm{A}}^{2} N_{\mathrm{A}}+\epsilon_{\mathrm{A}} \epsilon_{\mathrm{B}} N_{\mathrm{A}} C_{\mathrm{B}} h_{\mathrm{AB}}(k), \\
I_{\mathrm{B}}=\epsilon_{\mathrm{B}}^{2} N_{\mathrm{B}}\left[1+C_{\mathrm{B}} h_{\mathrm{BB}}(k)\right]+\epsilon_{\mathrm{A}} \epsilon_{\mathrm{B}} N_{\mathrm{B}} C_{\mathrm{A}} h_{\mathrm{AB}}(k), \\
I_{\mathrm{AB}}=\epsilon_{\mathrm{A}} \epsilon_{\mathrm{B}} N_{\mathrm{A}}+\epsilon_{\mathrm{B}}^{2} N_{\mathrm{A}} C_{\mathrm{B}} h_{\mathrm{AB}}(k), \\
D_{\mathrm{A}}=\frac{k_{\mathrm{B}} T}{f_{\mathrm{A}}}, \\
D_{\mathrm{B}}=\frac{K_{\mathrm{B}} T\left[1+C_{\mathrm{B}} h_{\mathrm{BB}}(k)\right]}{f_{\mathrm{B}}}, \\
D_{\mathrm{BA}}=\frac{C_{\mathrm{B}} h_{\mathrm{AB}} K_{\mathrm{B}} T}{f_{\mathrm{B}}} .
\end{gathered}
$$

Reference 25 shows that $D_{\mathrm{A}}$ is the tracer (self) diffusion coefficient of $\mathrm{A}$ in the mixture while $D_{\mathrm{B}}$ is the mutual diffusion coefficient of species $B$.

\section{APPENDIX B: DIFFUSION COEFFICIENT OF ONE COMPONENT OF A MIXTURE}

Our objective is to obtain the diffusion coefficient $D_{A}$ of a highly dilute species $A$ in the presence of a concentrated background of a second species B; both species are charged spherical particles. For simplicity, the ef fect of Debye-screened electrostatic interactions will be approximated by treating the particles as neutral hard spheres whose hydrodynamic radii $a_{H}$ are consistently less than their exclusion radii $a_{v}$. That is, the electrical forces keep the spheres touching each other. The initial slope of the dynamic structure factor of the A particles, neglecting interactions between pairs of A particles, gives $D_{\mathrm{A}}$ as
$-D_{\mathbf{A}} k^{2} S_{\mathbf{\Lambda}}(k, 0)=\lim _{t \rightarrow 0} \frac{d}{d t}\left\langle\frac{1}{N_{\mathrm{A}}} \sum_{t, t=1}^{N_{\mathrm{A}}} \exp \left\{i \mathbf{k} \cdot\left[\mathbf{r}_{j}(0)-\mathbf{r}_{\mathbf{t}}(t)\right]\right\}\right\rangle$,

$r_{i}(t)$ being the location of the $i$ th of the $N_{\mathrm{A}}$ A particles.

Equation (B1) is evaluated to first order in concentration by following Ref. 22. Dividing particle velocities into their Brownian and interactive parts, $v_{i}=v_{B i}+v_{I i}$, in the small-time limit $t \rightarrow 0, \mathrm{Eq}$. (B1) becomes

$$
\begin{aligned}
& -D_{\mathrm{A}} k^{2} S_{\mathrm{AA}}(k, 0)=\frac{1}{N_{\mathrm{A}}}\left\langle\sum _ { i , j = 1 } ^ { N _ { \mathrm { A } } } \operatorname { e x p } [ i \mathrm { k } \cdot \mathrm { r } _ { j i } ( 0 ) ] \left\{-i \mathrm{k} \cdot \mathrm{v}_{i}(t)\right.\right. \\
& \left.\left.-\int_{0}^{t-0} d s\left[\mathrm{k} \cdot \mathrm{v}_{B i}(s) \mathbf{k} \cdot \mathrm{v}_{B i}(t)+\mathbf{k} \cdot \mathrm{v}_{B i}(s) \mathbf{k} \cdot \mathrm{v}_{I i}(t)\right]\right\}\right\rangle .
\end{aligned}
$$

Comparison with Ref. 22, Eqs. (4.1)-(4.4), and use $e^{27}$ of Mazur's hydrodynamic interaction tensors for a pair of spheres of radii $a_{i}$ and $a_{l}$, separated by $r_{i l}$,

$$
\begin{aligned}
& \mu_{i t}=\frac{1}{6 \pi \eta a_{i}}\left[1-\frac{15}{4} \frac{a_{i} a_{l}^{3}}{r_{i l}^{4}} \hat{\mathbf{r}}_{i l} \hat{\mathbf{r}}_{i l}\right] \text {, } \\
& \mu_{i l}=\frac{1}{6 \pi \eta a_{i}}\left[\frac{3}{4} \frac{a_{i}}{r_{1 l}}\left(\mathrm{l}+\hat{\mathrm{r}}_{i l} \hat{\mathrm{r}}_{i l}\right)+\frac{a_{i}^{3}+a_{i} a_{l}^{2}}{4 r_{i l}^{3}}\left(\mathrm{t}-3 \hat{\mathrm{r}}_{i l} \hat{\mathrm{r}}_{i l}\right)\right],
\end{aligned}
$$

lets one write

$$
\begin{aligned}
& i \mathrm{k} \cdot \mathrm{v}_{i f}=\frac{i \mathrm{k}}{f_{i}}\left[1-\frac{15}{4} \sum_{i=1}^{N} \frac{a_{i} a_{i}^{3} \hat{\mathbf{r}}_{1 i} \hat{\mathbf{r}}_{1 l}}{r_{11}{ }^{4}}\right] \cdot F_{i}+\frac{i \mathrm{k}}{f}
\end{aligned}
$$

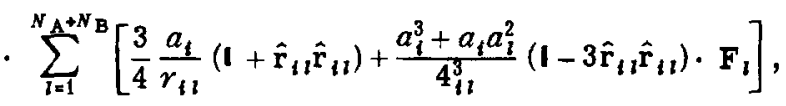

$$
\begin{aligned}
& \int_{0}^{t-0} \mathrm{k} \cdot \mathrm{v}_{B i}(s) \mathrm{k} \cdot \mathrm{v}_{B i}(t) d s=\frac{k_{B} T}{f_{i}}\left[1-\frac{15}{4} \sum_{i=1}^{N_{\mathrm{A}}+N_{B}} \frac{a_{i} a_{i}^{3}}{r_{i l}^{4}}\right] k^{2},
\end{aligned}
$$

where $f_{i}=6 \pi \eta a_{i}$ and $F_{i}$ is the direct force on particle $i$. The $\int \mathrm{v}_{B i}(s) \mathrm{v}_{I t}(t) d s$ term, which causes dynamic friction, is discussed in Sec. IV of this paper.

Equations (B2)-(B4) are combined and regrouped to separate terms which only depend on the presence of the A particles from terms which arise from the $B$ particles. Since species $\mathrm{A}$ is highly dilute, all $\mathrm{A}-\mathrm{A}$ interactions are set to zero [so $\left.S_{\mathrm{AA}}(k, 0)=1\right]$. The $\mathrm{A}-\mathrm{B}$ radial dis tribution function is approximated as

$$
\begin{array}{ll}
g_{\mathrm{AB}}(r)=0, & r<\sigma_{\mathrm{A}}+\sigma_{\mathrm{B}}, \\
g_{\mathrm{AB}}(r)=1, & r \geq \sigma_{\mathrm{A}}+\sigma_{\mathrm{B}},
\end{array}
$$

where $\sigma_{\mathrm{A}}>a_{\mathrm{B}}$ and $\sigma_{\mathrm{B}}>a_{\mathrm{B}}$. To first order in $C_{\mathrm{B}}$, the only effect of the B particles on $D_{\mathrm{A}}$ is through Eq. (BAb). Equation (B4a) vanishes because each term either involves the negligible $A-A$ forces or cancels by spherical symmetry. The remaining integral shows

$$
D_{\mathrm{A}}=D_{\mathrm{A}}^{0}\left(1-\frac{15}{4} \phi_{\mathrm{B}}\left[\frac{a_{\mathrm{A}}}{\sigma_{\mathrm{A}}+\sigma_{\mathrm{B}}}\right]\right)-\int \hat{\mathbf{k}} \cdot \mathrm{v}_{B i}(s) \hat{\mathbf{k}} \cdot \mathrm{v}_{I i}(t) d s .
$$

Here $\phi_{B}$ is the hydrodynamic volume fraction of $B$ particles, $D_{A}^{0}$ is the diffusion coefficient of the A particles 
in the absence of the $B$ particles and $k$ is a unit vector. Equation (B6), less the dynamic friction integral, is Eq. (5).

${ }^{1}$ P. N. Pusey, D. W. Schaeffer, D. E. Koppel, R. D. Camerini-Otero, and R. M. Franklin, J. Phys. (Paris) 33, C1-163 (1972).

${ }^{2}$ P. Doherty and G. B. Benedek, J. Chem. Phys. 61, 5426 (1974).

${ }^{3}$ G. D. J. Phillies, J. Chem. Phys. 60, 974 (1974).

${ }^{4}$ P. N. Pusey, J. Phys. A 8, 1433 (1975).

${ }^{5}$ B. J. Berne, in Photon Correlation Spectroscopy and Velocimitry, edited by H. Z. Cummins and E. R. Pike (Plenum, New York, 1977).

${ }^{6}$ S. S. Alpert and G. Banks, Biophys. Chem. 4, 287 (1976).

${ }^{7}$ G. D. J. Phillies, N. M. Mazer, and G. B. Benedek, J. Chem. Phys. 65, 1883 (1976).

${ }^{8}$ R. S. Hall, Y. S. Oh, and C. S. Johnson, Jr., J. Phys. Chem. 84, 756 (1980).

${ }^{9}$ P. N. Pusey, J. Phys. A 11, 119 (1973).

${ }^{10} \mathrm{P}$. S. Dalberg, A. Bळe, K. A. Strand, and T. Sikkeland, J. Chem. Phys. 69, 5473 (1978).

${ }^{11} \mathrm{~F}$. Gruner and W. Lehmann, in Light Scattering in Liquids and Macromolecular Solutions, edited by V. DeGiorgio, M, Corti, and M. Giglio (Plenum, New York, 1982).

${ }^{12} \mathrm{~W}$. Hess and R. Klein, Physica 94A, 71 (1978).

${ }^{13}$ G. D. J. Phillies, J. Chem. Phys. 74, 2436 (1981); Chem.
Phys. 74, 197 (1983).

${ }^{14}$ G. D. J. Phillies, Macromolecules 9, 447 (1976).

${ }^{15}$ M. B. Weissman, J. Chem. Phys, 72, 231 (1980).

${ }^{16} \mathrm{P}$. N. Pusey, in Ref. 11.

${ }^{17}$ G. D. J. Phillies, J. Chem. Phys. 74, 260 (1981); Phys. Rev. A 24, 1939 (1981).

${ }^{18}$ W. Hess and R. Klein, J. Phys. A 13 , L5 (1980).

${ }^{19}$ B. J. Ackerson, J. Chem. Phys. 64, 242 (1976).

${ }^{20}$ G. D. J. Phillies and P. R. Wills, J. Chem. Phys. 75, 508 (1981).

${ }^{21}$ C. W. J. Beenakker and P. Mazur, Phys. Lett. 91, 290 (1982).

${ }^{22}$ G. D. J. Phillies, J. Chem. Phys. 77, 2623 (1982).

${ }^{23}$ W. Hess, in Ref. 11 .

${ }^{24}$ P. N. Pusey, H. M. Fijnaut, and A. Vrij, J. Chem. Phys. 77, 4270 (1982).

${ }^{25}$ G. D. J. Phillies, J. Chem. Phys. 60, 983 (1974).

${ }^{26}$ G. D. J. Phillies, Biopolymers 14, 499 (1975).

${ }^{27} \mathrm{P}$. Mazur and W. van Saarloos, Physica A (in press).

${ }^{28}$ W. Hess and R. Klein, J. Phys. A 15, L669 (1982).

${ }^{2 \theta}$ (a) M. M. Kops-Werkhoven and H. M. Fijnaut, J. Chem. Phys. 74, 1618 (1981); (b) M. M. Kops-Werkhoven, H. J. Mos, P. N. Pusey, and H. M. Fijnaut, Chem. Phys. Lett. 81, 365 (1981); (c) M. M. Kops-Werkhoven and H. M. Fijnaut, J. Chem. Phys. 77, 2242 (1982); (d) M. M. KopsWerkhoven, C. Pathmamanoharan, A. Vrij, and H. M. Fijnaut, ibid. 77, 5913 (1982); (e) M. M. Kops-Werkhoven, A. Vrij, and H. N. W. Lekkerkerker, ibid. 78, 2760 (1983). ${ }^{30}$ J. G. Kirkwood, R. L. Baldwin, P. J. Dunlap, L. J. Gosting, and G. Kegeles, J. Chem. Phys. 33, 1505 (1960). 